\title{
CORRECTIONS AND SUPPLEMENTARIES TO MY PAPER CONCERNING KRULL-REMAK-SCHMIDT'S THEOREM
}

\author{
GORÔ AZUMAYA
}

1. It has recently been found that my previous paper "On generalized semiprimary rings and Krull-Remak-Schmidt's theorem," Jap. Journ. Math. 19 (1949) - referred to as S. K. - contained in its Theorems 19 and 20 some errors. Nevertheless the writer has been able to correct them in suitable forms so that most parts of both theorems hold, even under a weaker assumption, and also subsequent theorems remain valid. These will be, together with some supplementary remarks, shown in the present note. ${ }^{1)}$

For completeness let us recall several definitions. Let $R$ be any (associative) ring. An element $c$ of $R$ is called a root element if there exists no non-zero element $x$ such that $x a x=x$, or what comes to the same, if the left icieal $R c$, or equivalently, the right ideal $c R$ contains no non-zero idempotent element. We denote by $C$ the set of all root elements of $R$. Then in order that $C$ forms a two-sided ideal it is sufficient that $C$ is additively closed, that is, the sum of any two root elements is also root element. And, when this is the case, we say that $R$ possesses the radical $C . R$ is called semi-primary if $R$ possesses a radical (not identical with $R$ ) and every non-zero idempotent element contains a primitive idempotent element; if moreover all primitive idempotent elements are isomorphic to each other we call $R$ primary. $R$ is said to be completely primary when $R$ possesses a radical (again not identical with $R$ ) and every nonzero idempotent element is primitive.

Now suppose that every non-zero idempotent element in $R$ contains a primitive idempotent element. Then $R$ possesses a radical (i.e. $R$ is semi-primary) if and only if for every primitive idempotent element e the subring eRe possesses a rudical (i.e. $e$ Re is compleiely primary). For the proof we have only to prove the "if" part, since $e \mathrm{C} e=e \operatorname{Re} \cap \mathrm{C}$ is the set of all root elements of $e R e,{ }^{2)}$ and we may assume that $e R e$ possesses the radical $e C e$. Suppose that there were two root elements $a, b$ such that $a+b$ is not a root element (of $R$ ). Then $R(a+b)$

Received February 15, 1950.

1) We take this opportunity to correct the following errata: in the sixth line following the proof of Theorem 16 (page 537) both $\Re$ should be replaced by $\mathfrak{R}$.

2) S. K. Lemma 6. 
contains a non-zero idempotent element $e$, which we may by our hypothesis assume to be primitive. Then $e$ is expressed in a form $x(a+b)$ whence $e=e x(a+b) e=e x a e+e x b e$, and this is contrary to our assumption (that $e R e$ possesses the radical $e C e$ ). It is further to be noted that in case $R$ has a unit element $R$ is completely primary if and only if the sum of any two non-regular elements is always non-regular, and indeed, when this is the case, root elements are nothing but non-regular elements.)

2. Let $\mathfrak{M}$ be a module (with operator-domain) and let $R$ be its (operator-)endomorphism ring. Suppose that $\mathfrak{M}$ is decomposed into a (finite or infinite) direct sum of directly indecomposable (allowable) submoduli $\mathfrak{m}_{\mu}(\mu \in M)$ :

$$
\mathfrak{M}=\sum_{\mu \in M} \mathfrak{m}_{\mu} \text {. }
$$

Then there correspond mutually orthogonal primitive idempotent elements $e_{\mu}$ in $R$ such that $\mathfrak{M} e_{\mu}=\mathfrak{m}_{\mu}$, and $e_{\mu} R e_{\mu}$ may be considered, as usual, as the endomorphism ring of $\mathfrak{m}_{\mu}$. Let us denote by $M(\mu)$ the set of all indices $\kappa \in M$ such that $m_{k}$ is (operator-) isomorphic to $m_{\mu}$.

Throughout the following we assume that each $\mathfrak{m}_{\mu}$ satisfies the following condition:

(*) (4) The sum of any two proper endomorphisms ${ }^{5)}$ of $m_{\mu}$ is also proper, i.e. the endomorphism ring $e_{\mu} R e_{\mu}$ of $\mathrm{m}_{\mu}$ is completely primary.

LEMMA 1. Under the assumption (*), let $a$ and $b$ be two elements of $R$ such that $a+b=1$, the identity endomorphism. Then for any finite subset $\left\{\mu_{i}\right\}$ $=\left\{\mu_{1}, \mu_{2}, \ldots, \mu_{s}\right\}$ of $M$ we can find submoduli $\overline{\mathrm{m}}_{1}, \overline{\mathrm{m}}_{2}, \ldots, \overline{\mathrm{m}}_{s}$ so that each $\mathrm{m}_{\mu_{i}}$ is mapped isomorphically upon $\overline{\mathrm{m}}_{i}$, by means of either $a$ or $b$, and there holds the direct decomposition

$$
\mathfrak{M}=\overline{\mathfrak{m}}_{1}+\overline{\mathfrak{m}}_{2}+\ldots+\overline{\mathfrak{m}}_{s}+\sum_{\mu \in\left\{\mu_{i}\right\}} \mathfrak{m}_{\mu} .
$$

Proof. $a e_{\mu_{1}}$ and $b e_{\mu_{1}}$ induce on $m_{\mu_{1}}$ two endomorphisms whose sum is the identity. In virtue of our assumption $\left(^{*}\right)$, either $a e_{\mu_{1}}$ or $b e_{\mu_{1}}$ must induce an automorphism on $m_{\mu_{1}}$, or what is the same, $m_{\mu_{1}}$ is mapped by $a$ or $b$ isomorphically upon a submodule $\bar{m}_{1}$ and $\bar{m}_{1}$ is by $e_{\mu_{1}}$ carried isomorphically onto $m_{\mu_{1}}$. And the latter fact means, since $\sum_{\mu \neq \mu_{1}} m_{\mu}$ is the kernel of $e_{\mu_{1}}$, the following direct decomposability

3) Cf. Corollary 1 to Theorem 15; there the assumption that the unit element is the only non-zero idempotent element is superfluous; it follows automatically.

4) $=$ condition B) in S. K. Theorem 19.

5) $=$ non-automorphisms $=$ non-regular elements in the endomorphism ring of $\mathfrak{m}_{\mu}$. 


$$
\mathfrak{M}=\overline{\mathfrak{m}}_{1}+\sum_{\mu \neq \mu_{1}} m_{\mu} \text {. }
$$

Apply next the above consideration to this direct decomposition and $m_{\mu_{2}}$ (in place of the given direct decomposition (1) and $m_{i_{1}}$ ). Then we obtain a second submodule $\bar{m}_{2}$; observe that $\bar{m}_{1}$ fulfills, with $m_{\mu_{1}}$, the same assumption as $(*)$. Continuing in this way we have the required submoduli.

Now we prove, as an improvement as well as a correction of $\mathrm{S}$. K. Theorem 19, the following

THEOREM 1.: Lei the dired decomposition (1) satisfy the condition (*) above. Then:

i) For chy non-kero idempolent element $f$ in $R$, there exists at least one mu such that $f$ an isomernhism on mu and the isomorphic image $m_{\mu} f$ is a (direcily indecomposable) direct simmand of Mi. In particular, pvery directly indecomposable direct summant is isomorphic to one of mp's.

ii) Given a second direct decomposition of $\mathrm{M}$ inio directly indecomposable submoduli u, $(y \in N)$ :

$$
\mathfrak{M}=\sum_{\nu \in \mathrm{N}} \mathfrak{n}_{\nu}
$$

then there exists a one-to-one mapping $\mu \rightarrow \nu(\mu)$ of $M$ onto $N$ such that $m_{\mu}$ is isomorphic to $n_{x(k)}$, for each $\mu \in M$; or, in other words, the direct decomposition of $\mathfrak{M}$ inio directiy indecomposable submoduli is unique up to automorphisms.

Proof. i) If we put $f^{\prime}=1-f, f^{\prime}$ is idempotent and is orthogonal to $f$ and $\mathfrak{M}$ is the direct sum of $\mathfrak{M} f$ and $\mathfrak{M} f^{\prime}$. Let $v$ be any non-zero element from $\mathfrak{M} f$ and let $\mu_{1}, \mu_{2}, \ldots, \mu_{s}$ be the finite number of indices such that $v e_{\mu_{i}} \neq 0$ for $i=1,2, \ldots, s$ and $v e_{\mu}=0$ whenever $\mu \notin\left\{\mu_{i}\right\}$. Then, on applying the preceding lemma to $a=f, b=f^{\prime}$ and $\mu_{1}, \mu_{2}, \ldots, \mu_{s}$, we have directly indecomposable direct summands $\bar{m}_{1}, \bar{m}_{2}, \ldots, \bar{m}_{s}$ of $\mathfrak{M}$ such that the module sum $\overline{\mathfrak{m}}_{1}+\overline{\mathfrak{m}}_{2}+\ldots$ $+\bar{m}_{s}$ is direct, each $\bar{m}_{i}$ is isomorphic to $m_{\mu_{i}}$ and in fact the isomorphism is induced by $f$ or $f^{\prime}$. Suppose that this were the case for every $i$ exclusively by $f^{\prime}$. Then $f^{\prime}$ would nap $m_{\mu_{1}}+m_{\mu_{2}}+\ldots+m_{\mu_{s}}$ isomorphically upon $\bar{m}_{1}+\bar{m}_{2}+\ldots$ $+\overline{\mathrm{m}}_{s}$, and this is a contradiction, since $v \neq 0, v f^{\prime}=0$ and $v=v e_{\mu_{1}}+v e_{\mu_{2}}+\ldots$ $+v e_{\mu_{s}}$ is contained in $m_{\mu_{1}}+m_{\mu_{2}}+\ldots+m_{\mu_{3}}$. Thus at least one $m_{\mu_{l}}$ is mapped by means of $f$ isomorphically onto $\overline{\mathfrak{m}}_{i}=\mathfrak{m}_{\mu_{i}} f$ 。

ii) Each $n_{\nu}$ is, by i), isomorphic to some $m_{\mu}$, and consequently the direct decomposition (2) also satisfies the same condition as $\left(^{*}\right)$. Hence we may, by

B) This theorem can readily be transferred, together with Lemma 1 , to non-commutative groups if we consider "normal endomorphisms" and make use of the notion of their "Addierbarkeit." Cf. footnote (27) in S. K. 
interchanging (1) and (2), conclude similarly that each $m_{\mu}$ is isomorphic to some $\mathfrak{n}_{\nu}$. As $M(\mu)$, we denote by $N(\nu), \nu$ being in $N$, the set of all $\lambda \in N$ such that $\mathfrak{n}_{\lambda}$ is isomorphic with $\mathfrak{n}_{\nu}$. Then our assertion means that if $\mathfrak{m}_{\mu}$ and $\mathfrak{n}_{\nu}$ are isomorphic $M(\mu)$ and $N(\nu)$ have the same cardinal number: $\overline{\overline{M(\mu}})=\overline{\overline{N(\nu)}}$; and to prove this we have, by symmetry, only to show that $\overline{\overline{M(\mu)}} \supseteqq \overline{\overline{N(\nu)}}$.

Changing letters for convenience, let us assume that $\mathfrak{m}_{\kappa}$ and $\mathfrak{n}_{\lambda}$ are isomorphic. Let $f_{\nu}$ be, for each $\nu \in N$, the primitive idempotent element belonging to $\mathfrak{n}_{2}$ (with respect to the direct decomposition (2)). Consider any index $\nu_{0}$ from $N(\lambda)$. Then, on applying i) to $f=f_{\nu_{0}}$, we can find an index $\mu_{0} \in M(\kappa)$ so that $\mathfrak{m}_{\mu_{0}}$ is carried by $f_{\nu_{0}}$ isomorphically onto $\mathfrak{n}_{\nu_{0}}$ (since $\mathfrak{M} f_{\nu_{0}}=\mathfrak{n}_{\nu_{0}}$ is directly indecomposable); we have the direct decomposition

$$
\mathfrak{M}=\mathfrak{m}_{\mu_{0}}+\sum_{\nu \neq \nu_{0}} \mathfrak{n}_{\nu}
$$

Now let $\nu_{1}, \nu_{2}, \ldots, \nu_{s}$ be any finite number of indices from $N(\lambda)$. Then, on repeating the above argument $s$-times in the similar manner as in the proof of Lemma 1 , we can readily show the existence of $s$ indices $\mu_{1}, \mu_{2}, \ldots, \mu_{s}$ in $M(\kappa)$ for which the direct decomposition

$$
\mathfrak{M}=\mathfrak{m}_{\mu_{1}}+\mathfrak{m}_{\mu_{2}}+\ldots+\mathfrak{m}_{\mu_{s}}+\sum_{\nu \in(\nu i\}} \mathfrak{n}_{\nu}
$$

holds. From this follows in particular that if $M(\kappa)$ is finite then $N(\lambda)$ is also finite and $\overline{\bar{M}(\kappa)} \equiv \overline{\bar{N}} \overline{\overline{(\lambda)}}$.

We assume therefore that $M(\kappa)$ is infinite. Consider any index $\mu$ from $M(\kappa)$. Then, since for an arbitrary non-zero element $u$ from $\mathfrak{m}_{\mu}$ the $\mathfrak{n}_{\nu}$-component $u f_{\nu}$ vanishes for almost all $\nu$, there exist all the more only finitely many indices $\nu \in N(\lambda)$ such that $\mathrm{m}_{\mu}$ is by means of $f_{\nu}$ mapped isomorphically upon $\mathfrak{n}_{\nu}$. We denote this finite subset of $N(\lambda)$ by $F(\mu)$, which we associate with each $\mu$. Then, as was shown above, if $\mu$ runs over all indices in $M(\kappa)$ the corresponding $F(\mu)$ exhaust $N(\lambda)$ :

$$
\bigcup_{\mu \in M(\kappa)} F(\mu)=N(\lambda) .
$$

This implies, since $M(\kappa)$ is infinite, that $\overline{\overline{M(\kappa)}} \equiv \overline{\overline{N(\lambda)}}$, and the proof is completed.

3. Now we verify the following theorem, the first part i) of which may be seen as an improvement of S. K. Theorem 20, i).

Theorem 2. Under the assumption (*) above:

i) The endomorphism ring $R$ of $\mathfrak{M}$ is semi-primary, and every primitive idempotent element in $R$ is isomorphic to one of $e_{\mu}$ 's. 
ii) Every element $a$ of $R$ such that $a \equiv 1$ (mod. $C$ ), $C$ being the radical of $R$, is an isomorphism of $\mathfrak{M}$ (into itself), and for any finite number of indices $\mu_{1}, \mu_{2}, \ldots, \mu_{s}$ from $M$ we have the following direct decomposition:

$$
\mathfrak{M}=\mathfrak{m}_{\mu_{1}} a+\mathfrak{m}_{\mu_{2}} a+\ldots+\mathfrak{m}_{\mu_{s}} a+\sum_{\mu \in\left(\mu_{i}\right)} m_{\mu} .
$$

Proof. i) is an immediate consequence of Theorem 1, i). Indeed, that $\mathfrak{M} f$ contains a directly indecomposable direct summand $m_{\mu} f$ isomorphic to $m_{\mu}$ means that $f$ contains a primitive idempotent element $e$ isomorphic to $e_{\mu}$ such that $\mathfrak{M} e=\mathrm{m}_{\mu} f$; further, in this case, the subring $e$ Re pussesses a radical, since $e_{\mu} R e_{\mu}$ does the same by the assumption $\left(^{*}\right)$ and is isomorphic with $e R e$. These show, as was remarked in $\S 1$, that $R$ is semi-primary.

ii) By virtue of Lemma 1 , it is sufficient to prove that if we put $b=1-a$ no non-zero direct summand can be mapped isomorphically by means of $b$ onto a direct summand. Suppose contrarily that there were two direct summands $\mathfrak{m}=\mathfrak{M} e$ and $\mathfrak{n}=\mathfrak{M} f$, where both $e$ and $f$ are non-zero idempotent elements in $R$, such that $b$ carries $m$ isomorphically onto $\mathfrak{n}$. Then the isomorphism would have an inverse isomorphism $x \in f R e$, which fulfills obviously the rèlation $x b$ $=f$. But this is contrary to our assumption that $b=1-a$ is in $C$.

We can further show that Corollary 2 to S. K. Theorem 19 also holds under the assumption $(*)$ alone:

Lemma 2.7) Let the direct decomposition (1) satisfy the assumption (*). Then for any given primitive idempotent elemen: $e$ in $R$ there exists at least one $\mu$ such that $e_{\mu} e e_{\mu}$ is an automorphism on $\mathrm{m}_{\mu}$.

Proof. If we put $m=\mathfrak{M} e, m$ is, according to Theorem $1, \mathrm{i}$ ), isomorphic to one of $m_{\mu}$ 's and hence satisfies the same assumption as $\left(^{*}\right)$. Now each $e_{\mu} e$ induces on $\mathrm{m}$ an endomorphism $e e_{\mu} e$. The totality $\left\{e e_{\mu} e ; \mu \in M\right\}$ is summable with respect to $m$ and the sum $\sum_{\mu} e e_{\mu} e=e$ is the identity endomorphism on $m$. Let $u$ be an arbitrary non-zero element of $m$ and let $\mu_{1}, \mu_{2}, \ldots, \mu_{s}$ be a finite number of indices such that $u e e_{\mu} e\left(=u e_{\mu} e\right)=0$ whenever $\mu \notin\left\{\mu_{i}\right\}=\left\{\mu_{1}, \mu_{2}\right.$, $\left.\ldots, \mu_{s}\right\}$. Then the sum $d=\sum_{\mu \notin\left\{\mu_{i}\right\}} e e_{\mu} e$ is, since $u d=0$, a proper endomorphism on $m$ and satisfies $e e_{\mu_{1}} e+e e_{\mu_{2}} e+\ldots+e e_{\mu_{s}} e+d=e$. From this follows, by ${ }^{*}$ ) for $\mathrm{m}$, that ${ }^{8)}$ there must exist at least one $\mu_{i}$ such that $e e_{\mu_{i}} e$ is an automor-

7) The validity of this lemma and that of the succeeding theorem under the assumption (*) alone were communicated to the writer by T. Nakayama.

8) Generally, if $\mathfrak{m}$ is a directly indecomposable module satisfying the same assumption as (*) and if $\left\{a_{0}\right\}$ be a summable system of proper endomorphisms of $m$ then the sum $\sum_{\sigma} a_{\sigma}$ is also proper. 
phism on $\mathrm{m}$, or what is the same, $e_{\mu_{i}}$ and $e$ map $\mathrm{m}$ and $\boldsymbol{m}_{\mu_{i}}$ isomorphically upon $\boldsymbol{m}_{\mu_{i}}$ and $m$ respectively. $\mathfrak{D}_{i}$ is therefore the direct sum of $\mathfrak{m}_{\mu_{\mu_{i}}}$ and the kernel $\mathfrak{M}(1-e)$ of $e$, and $\mathfrak{m} e_{\mu_{i}}$ is a direct summand. But since $m e_{\mu_{i}}$ is contained in the directly indecomposable module $\mathfrak{m}_{\mu_{i}}\left(=\mathfrak{M} e_{\mu_{i}}\right)$, we have necessarily $\mathfrak{m}_{\mu_{i}}$ $=m_{\mu_{i}}$. Thus $e_{\mu_{i}} e e_{\mu_{i}}$ is an automorphism on $m_{\mu_{i}}$.

By virtue of the preceding lemma, we obtain the following

THEOREM 3.") Under the assumption (*) above:

i) The radical $C$ of $R$ consists of all elements $c$ in $R$ such that $e_{\mu} c e$ is, for every $\mu, \nu \in M$, a proper honomorphism of $\mathrm{m}_{\mu}$ into $\mathrm{m}_{\mathrm{\nu}}$.

ii) The residue class ring $\bar{R}=R / C$ modulo the radical $C$ is a semi-irreducible resular ring and may be considered as the endomorphism ring of the left

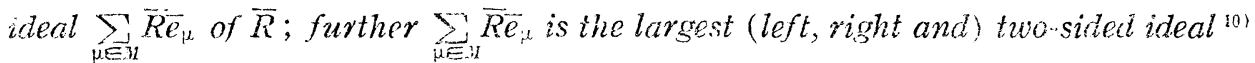
of $\bar{R}$.

Proof. i) That if $c$ is in $C$ then each $e_{i 2} C_{2}$ is a proper homomorphism of $u u_{k}$ into $m_{2}$ can be proved in the same way as in the proof of Theorem 2, ii), since $e_{y} c e_{\nu}$ also in $C$. Suppose contrarily that an element $d$ of $R$ is not in $C$. Then the right ideal $d R$ contains a non-zero idempotent element $e=d x$, which we may, since $R$ is semi-primary by Theorem 2, assume to be primitive. According to Lemma 2, there exists an index $\mu$ such that $e_{\mu} e e_{\mu}=e_{\mu} d x e_{\mu}$ is an automorphism on $m_{\mu}$. Consider now a summable system $\left\{e_{\mu} d e_{\nu} x e_{\mu} ; \nu \in M\right\}$ of endomorphisms of $m_{j \mu}$. Then it has the sum $\sum_{\nu} e_{\mu} d e_{\nu} x e_{i L}=e_{i L} d x e_{\mu}$ and hence there must exist, ${ }^{\prime \prime \prime}$ by the assumption (*), at least one $\nu$ such that $e_{i 2} d e_{i} x e_{\mu}$ is an automorphism on $m_{\mu}$. Then $e_{\mu} d e_{\nu}$ maps $m_{\mu}$ isomorphically onto $m_{\nu}$, that is, $e_{\mu} d e_{\nu}$ is not a proper homomorphism of $m_{\mu}$ into $m_{\nu}$.

ii) Let $a$ be any element of $R$. For each $\mu, e_{\mu} a e_{\nu}$ is a proper homomorphism. of $m_{\mu}$ into $m_{\nu}$ except for a finite number of indices $\nu_{,}{ }^{12)}$ say, $z^{\prime}=\nu_{1}, \nu_{2}, \ldots, \nu_{3}$. Then it can readily be seen from i) of this theorem that $e_{\mu} a-\left(e_{\mu} a e_{\gamma_{1}}+e_{\mu} a e_{\gamma_{3}}\right.$ $\left.+\ldots+e_{\mu} a e_{v_{s}}\right)$ lies in the radical $C$, i.e. $\bar{e}_{\mu} \bar{a}=\bar{e}_{\mu} \bar{a} \bar{e}_{\nu_{1}}+\bar{e}_{k} \bar{a} \bar{e}_{\nu_{2}}+\ldots+\bar{e}_{\mu} \bar{a} \bar{e}_{\nu_{s}}$. This shows that $\sum_{\mu} \bar{R} \bar{e}_{\mu}$ is a two-sided ideal of $\bar{R}$. Every element of $\bar{R}$ induces therefore an (operator-)endomorphism of the left ideal $\sum_{\mu} \bar{R} \bar{e}_{\mu}$, and indeed distinct elements induce distinct endomorphisms. For, if $a$ is an element of $R$ such that $\bar{e}_{\mu} \bar{a}=\overline{0}$ for every $\mu$ then $\bar{e}_{\mu} \bar{a} \bar{e}_{\nu}=\overline{0}$, whence $e_{\mu} a e_{\nu}$ is a proper homo-

9) This theorem was given in S. K. Theorem $20, \mathrm{i}$ ) (in its proof. to be precise) and iii) by assuming not only the assumption (*) but also the assumption (**) below.

10) Cf. Corollary to S. K. Theorem 11.

11) Take account of footnote 8 ).

12) Because for an arbitrary non-zero element $u$ of $\mathfrak{m}_{\mu} u e_{\mu} a e_{2}$, vanishes for almost all $\nu$. 
morphism of $m_{\mu}$ into $m_{\nu}$ for every $\mu, \nu$, and consequently $a$ is in $C$ again by i) of this theorem. Now let there be given an endomorphism of $\sum_{\mu} \bar{R}_{\bar{e}_{\mu}}$ which makes correspond $\bar{e}_{\mu} \rightarrow \bar{a}_{i \mu}$. Since $\bar{e}_{\mu} \bar{a}_{\mu}=\bar{a}_{i \mu}$, we can take a representative $a_{\mu}$ of $\bar{a}_{\mu}$ so that $e_{\mu} a_{\mu}=a_{\mu}$. The system $\left\{a_{\mu} ; \mu \in M\right\}$ is then summable and if we put $a=\sum_{\mu} a_{\mu}$ we have $e_{\mu} a=e_{\mu} a_{\mu}=a_{\mu}$ whence $\bar{e}_{\mu} \bar{a}=\bar{a}_{\mu}$, which means that $\bar{a}$ induces the given endomorphism. Thus it is proved that $\bar{R}$ may be considered as the endomorphism ring of the left ideal $\sum_{\mu} \bar{R}_{e_{\mu}} . \quad \bar{R}$ is semi-irreducible by Corollary to S. K. Theorem 10, while $\bar{R}$ is regular by virtue of S. K. Theorem 16 because $\sum_{\mu} \bar{R} \bar{e}_{\mu}$ is completely reducible. Every simple left ideal of $\bar{R}$ is, since $\bar{R}$ is semiirreducible, expressed in the form $\bar{R} \bar{e}$ by a primitive idempotent element $\bar{e}$. Taking a primitive idempotent representative $e$ of $\bar{e},{ }^{131} e$ is by Theorem 2, i) isomorphic to some $e_{\mu}$, that is, $e \in R e_{\mu} R^{14)}$ whence $\bar{e} \in \bar{R} \bar{e}_{\alpha} \bar{R}$, which implies that (the simple left ideal) $\bar{R} \bar{e}$ is contained in $\sum_{\mu} \bar{R} \bar{e}_{\mu} \quad$ Thus $\sum_{\mu} \bar{R} \bar{e}_{\mu}$ is the largest completely reducible left (whence right as well as two-sided) ideal of $\bar{R}$.

We now impose, for the first time, the following assumption besides (*):

$\left(^{* *}\right)^{15}$ In case the cardinal number $\overline{\bar{M}}$ of $\left\{m_{\mu}\right\}$ is infinite, each $m_{\mu}$ is finitely generated.

We can readily see that under both the assumptions (*) and (**) S. K. Theorem 20, ii), whence the succeeding Theorems 21-23 also, remain valid with the same proofs as there. Whether or not S. K. Theorem 19, together with its Corollary 1, and S. K. Theorem 20, iv) hold valid under the assumptions (*) and $(* *)$, the writer has however to leave here open.

Remark. Although every element $a$ of $R$ such that $a \equiv 1$ (mod. $C$ ) is, according to Theorem 2, ii), an isomorphism of $\mathfrak{M}$ into itself, $a$ is not necessarily an automorphism, ${ }^{16)}$ as the following example shows:

Let $R_{0}$ be a completely primary ring with unit element whose radical $C_{0}$ is not a nil-ideal "ii) and let $R$ be the row-finite matrix ring over $R_{0}$ of countably infinite dimension, say. Then $R$ is regarded as an endomorphism ring of a countably infinite direct sum of (directly indecomposable) submoduli all isomorphic to $R_{0}$ (with the left operator-ring $R_{0}$ ), which fulfills certainly the assumptions (*) and (**), and moreover the radical $C$ of $R$ consists of all matrices in $R$ such that all its elements lie in $C_{0}{ }^{13)}$ Now let $c$ be any non-nilpotent element

\footnotetext{
13) Cf. S. K. Theorem 8.

14) Cf. S. K. Lemma 8.

15) $=$ condition A) in S. K. Theorem 19.

16) But in case $M$ is finite $a$ is always an automorphism, as Theorem 2, ii) shows.

1i) We may take $R_{0}$ to be a valution ring of a $p$-adic number field, for instance.

18) Cf. S. K. Theorem 22.
} 
from $C_{0}$ and consider the matrix

$$
a=\left(\begin{array}{rrrrrr}
1 & -c & & & & 0 \\
& 1 & -c & & & \\
& 1 & -c & & \\
& & \cdot & \cdot & \\
& & & \cdot & \cdot & \cdot
\end{array}\right)
$$

Then $a$ is in $R$ and $a \equiv 1(\bmod . C$ ), but $a$ is not regular in $R$. For, if $a$ could have a left inverse, it would be of the form

$$
\left(\begin{array}{ccccc}
1 & c & c^{2} & \ldots & \ldots \\
1 & c & c^{2} & \ldots & \ldots \\
& 1 & c & c^{2} & \ldots
\end{array}\right]
$$

but this matrix is not row-finite because of the non-nilpotency of $c$.

Department of Mathematics, Nagoya University 